\title{
Surgical resection or aspiration with ethanol sclerotherapy of endometrioma before in vitro fertilization in infertilie women with endometrioma
}

\author{
Kyung-Hee Lee, Chung-Hoon Kim, You-Jeong Lee, Sung-Hoon Kim, Hee-Dong Chae, Byung-Moon Kang \\ Division of Reproductive Endocrinology and Infertility, Department of Obstetrics and Gynecology, University of Ulsan College of Medicine, Asan \\ Medical Center, Seoul, Korea
}

\section{Objective}

To evaluate whether the surgical resection or aspiration with ethanol sclerotherapy (AEST) of endometrioma before in vitro fertilization (IVF) affect controlled ovarian stimulation (COS) and IVF outcome in the infertilie women with endometroma undergoing IVF.

\section{Methods}

In this retrospective cohort study, 101 consecutive IVF/intracytoplasmic sperm injection cycles that were performed in 101 patients with endometrioma(s) between January 2008 and December 2012 were included. Before IVF, 36 patients underwent surgical resection of endometrioma (resection group), 29 patients had transvaginal endometrioma AEST (aspiration group), and 36 patients did not take any surgical intervention (control group). The three groups were compared in terms of COS and IVF outcomes.

\section{Results}

Total antral follicle count was significantly lower in the resection group than in the aspiration or control group. The numbers of follicles with a diameter of 14 to $17 \mathrm{~mm}$ on the human chorionic gonadotropin day, retrieved oocytes, mature oocytes, and fertilized oocytes were significantly lower in the resection group than in two other groups. However, three groups were similar in terms of clinical pregnancy rate (CPR) per initiated cycle, CPR per embryo transfer, embryo implantation rate, and miscarriage rate.

\section{Conclusion}

Neither of surgical resection and AEST of endometrioma before IVF treatment can give any beneficial effect on IVF outcomes. Moreover, surgical resection of endometrioma can affect the ovarian reserve and ovarian response during $\cos$.

Keywords: Aspiration with ethanol sclerotherapy; Endometriosis; Fertilization in vitro; Surgical resection

\section{Introduction}

Endometriosis is one of the most common indications for assisted reproductive technologies (ARTs) because endometriosis can cause subfertility with anatomical damage and various inflammatory substances. Although the association between minimal or mild endometriosis and infertility is less clear, there is little debate that moderate or severe endometriosis can impair fertility.

There has been some evidence that the presence of ovarian endometrioma impairs the quality of oocyte as revealed
Received: 2013.11.29. Revised: 2014.1.2. Accepted: 2014.1.14. Corresponding author: Chung-Hoon Kim

Division of Reproductive Endocrinology and Infertility, Department of Obstetrics and Gynecology, University of Ulsan College of Medicine, Asan Medical Center, 88 Olympic-ro 43-gil, Songpa-gu, Seoul 138-736, Korea

Tel: +82-2-3010-3639 Fax: +82-2-3010-6944

E-mail: chnkim@amc.seoul.kr

Articles published in Obstet Gynecol Sci are open-access, distributed under the terms of the Creative Commons Attribution Non-Commercial License (http://creativecommons. org/licenses/by-nc/3.0/) which permits unrestricted non-commercial use, distribution, and reproduction in any medium, provided the original work is properly cited.

Copyright $\odot 2014$ Korean Society of Obstetrics and Gynecology 


\title{
Obstetrics \& Gynecology Science
}

\author{
Vol. 57, No. 4, 2014
}

by their fertilization and implantation ability [1-3]. However, the influences of ovarian endometrioma on the outcome of ART is still controversial and the optimal treatment of ovarian endometrioma before in vitro fertilization (IVF) treatment now open for debate. Recently, interventions for women with endometrioma prior to ART was investigated in Cochrane review analyzing 4 randomized controlled trials. They analyzed the efficacy of aspiration or cystectomy versus expectant management to find no evidence of a benefit for clinical pregnancy with either technique. However, they could not evaluate the live birth rate in their study and indicated that there is too much clinical and statistical heterogeneity between the trials to compare their results [4]. Moreover, there is limited data that compare the efficacy of the aspiration and surgical removal of endometrioma before ART in one study. Therefore, we performed the study to investigate the effect of surgical resection and transvaginal aspiration with ethanol sclerotherapy (AEST) of endometrioma before IVF on controlled ovarian stimulation (COS) and IVF outcome in the infertilie women with endometroma undergoing IVF.

\section{Materials and methods}

\section{Patients}

This retrospective cohort study included 101 consecutive IVF/ intracytoplasmic sperm injection (ICSI) cycles that were performed in 101 patients with endometrioma(s) between January 2008 and December 2012. Of 101 patients, 36 patients underwent surgical resection of endometrioma (resection group), 29 patients had transvaginal endometrioma AEST (aspiration group) before IVF/ICSI, and 36 patients did not take any surgical intervention (control group). This study was approved by the Institutional Review Board of Asan Medical Center, University of Ulsan College of Medicine (IRB number: 2013-1090). The clinical information was obtained by chart review.

The following selection criteria were adopted for this study: 1) women with pathologically confirmed endometrioma(s) previously (resection group, aspiration group) or with endometrioma(s) of a mean diameter of $>3 \mathrm{~cm}$ or diagnosed by transvaginal ultrasonography (control group); 2) women of 20 to 45 years old at the time of screening; 3 ) women with normal ovulatory cycles of 24 to 35 days in length; and 4) body mass index (BMI) between 18 and $25 \mathrm{~kg} / \mathrm{m}^{2}$.

Patients with current endocrine abnormalities such as dia- betes mellitus, polycystic ovary syndrome, hyperprolactinemia were excluded from this study. Patients who had any abnormalities that would interfere with adequate stimulation, previous hospitalization due to severe ovarian hyperstimulation syndrome, or a history of previous (within 12 months) or current abuse of alcohol or drugs were also excluded from the present study.

In the resection group, the patients who underwent IVF within 5 years from the surgical resection of endometrioma were included regardless of the type of COS protocol. We included the patients who had the resection of endometrioma only once and excluded the patients in whom fulguration of pelvic endometriosis or adhesiolysis without the resection of endometrioma was performed. All patients included in the resection group were pathologically confirmed as endometriosis. In the aspiration group, the patients who underwent IVF within 1 year from AEST of endometrioma were recruited regardless of the type of COS protocol. In the control group, the patients who had IVF without any intervention for endometrioma before IVF treatment were included. We excluded the patient with recurrent endometrioma having previous surgical resection of ovarian endometrioma from the aspiration or control group.

If patients underwent two or more cycles of IVF/ICSI during the study period, charts corresponding to the first IVF/ICSI cycle were reviewed and data of other IVF/ICSI cycles except first cycle were excluded from this analysis.

\section{Transvaginal aspiration with ethanol sclerotherapy of endometrioma}

Transvaginal AEST of endometrioma was performed as an outpatient procedure under intravenous sedation using propofol sodium. Patients were placed in the lithotomy position, and monitored using an electrocardiogram and a pulse oximeter. Under transvaginal ultrasound guidance, $35 \mathrm{~cm}$ long 18-gauge needle was inserted through the vaginal fornix into the endometrioma. The endometriotic cyst content was aspirated and flushed twice or three times with 20\% pure sterile ethanol (Merck Serono SA, Geneva, Switzerland) in a volume equal to $80 \%$ to $90 \%$ of the aspirated volume. The aspirated endometriotic fluid was sent for cytologic analysis.

\section{Controlled ovarian stimulation and in vitro fertilization treatment}

Gonadotropin releasing hormone $(\mathrm{GnRH})$ agonist long pro- 


\section{Obstetrics \& Gynecology Science}

Kyung-Hee Lee, et al. Intervention of endometrioma before IVF

tocol or GnRH antagonist protocol was used for COS in all subjects. In the GnRH agonist long protocol, daily injection of a GnRH agonist $1 \mathrm{mg}$ (Leuprorelin acetate, Lorelin, Dongkook, Seoul, Korea) was initiated during the midluteal phase of the preceding menstrual cycle and was continued at least for 14 days, followed by a dose reduction to $0.5 \mathrm{mg}$ daily. $\mathrm{GnRH}$ agonist $0.5 \mathrm{mg}$ was continued daily up to day of human chorionic gonadotropin (hCG) administration. Ovarian stimulation was started with 150 to 300 IU of recombinant human follicle-stimulating hormone (rhFSH; Gonal-F, Merck Serono SA) after establishing ovarian and uterine quiescence using vaginal ultrasound. The rhFSH dose was adjusted every 3 to 4 days according to the ovarian response. A recombinant hCG (rhCG; Ovidrel, Merck Serono SA) of $250 \mu \mathrm{g}$ was injected to induce follicular maturation when one or more follicles reached a mean diameter of $\geq 18 \mathrm{~mm}$. Oocyte retrieval (OR) was performed 35 to 36 hours after hCG injection, and 1 to 4 embryos were transferred into the uterus on the third day after OR. Vaginal progesterone gel (Crinone $8 \% 90 \mathrm{mg}$, Merck Serono SA) once daily was administrated from the day of OR for luteal support.

In the GnRH antagonist protocol, ovarian stimulation was commenced with 150 to $300 \mathrm{IU}$ of rhFSH, from the third day of menstruation after establishing ovarian and uterine quiescence by using transvaginal ultrasound. The rhFSH dose was adjusted every 3 to 4 days according to the ovarian response.
When the leading follicle reached 13 to $14 \mathrm{~mm}$ in an average diameter, GnRH antagonist cetrorelix (Cetrotide, Merck Serono $\mathrm{SA}$ ) at a dose of $0.25 \mathrm{mg} /$ day was started and continued daily up to the day of hCG injection. When one or more follicles reached a mean diameter of $18 \mathrm{~mm}$ or more, a single subcutaneous bolus of $250 \mu \mathrm{g}$ rhCG (Ovidrel, Merck Serono SA) was administered simultaneously with GnRH agonist $0.1 \mathrm{mg}$ (Decapeptyl, Ferring, Malmö, Sweden). OR and luteal support were performed in same manner with $\mathrm{GnRH}$ agonist long protocol.

After IVF or ICSI, one to four embryos were transferred into the uterus on the third day after OR. Starting on the day of $\mathrm{OR}$, all patients received $90 \mathrm{mg}$ of vaginal progesterone gel (Crinone gel 8\%, Merck Serono SA) once daily for luteal phase support. The $\beta$-hCG serum levels were measured 11 days after embryo transfer (ET). The $\beta$-hCG serum levels were measured by radioimmunoassay using a hCG MAIA clone kit (Serono Diagnostics, Woking, UK) with interassay and intraassay variances of $<10 \%$ and $5 \%$, respectively, 11 days after ET. Clinical pregnancy was defined as the presence of a gestational sac by ultrasonography, while miscarriage rate per clinical pregnancy was defined as the proportion of patients who failed to continue development before 20 weeks of gestation in all clinical pregnancies.

\section{Statistical analysis}

Mean values were expressed as mean \pm standard deviation.

Table 1. Patients' characteristics

\begin{tabular}{|c|c|c|c|c|}
\hline Characteristic & $\begin{array}{l}\text { Cystectomy group } \\
(n=36)\end{array}$ & AEST group $(n=29)$ & $\begin{array}{l}\text { Control group } \\
\quad(n=36)\end{array}$ & $P$-value \\
\hline Age of patients (yr) & $33.6 \pm 2.9$ & $34.6 \pm 4.9$ & $34.3 \pm 4.3$ & NS \\
\hline Age of husbands (yr) & $35.6 \pm 3.2$ & $36.7 \pm 4.5$ & $35.5 \pm 4.4$ & NS \\
\hline Infertility duration (mo) & $41.0 \pm 35.3$ & $45.6 \pm 29.1$ & $34.9 \pm 28.8$ & NS \\
\hline $\mathrm{BMI}\left(\mathrm{kg} / \mathrm{m}^{2}\right)$ & $21.9 \pm 2.1$ & $21.6 \pm 1.7$ & $21.6 \pm 1.7$ & NS \\
\hline No. of nullipara & $31(86.1)$ & $25(86.2)$ & $31(86.1)$ & NS \\
\hline AFC & $8.2 \pm 3.9^{\text {a) }}$ & $11.1 \pm 3.8$ & $11.2 \pm 4.7$ & 0.007 \\
\hline No. of patients with AFC $\leq 6$ & $18(50)^{b)}$ & $10(34.4)$ & $5(13.9)$ & 0.005 \\
\hline Duration between intervention and IVF (mo) & $20.3 \pm 19.5$ & $3.1 \pm 3.0$ & - & $<0.001$ \\
\hline Size of endometrioma before resection or AEST (mm) & $58.8 \pm 15.3$ & $51.5 \pm 18.2$ & - & NS \\
\hline Size of endometrioma on the day of OR (mm) & $22.6 \pm 24.1$ & $15.5 \pm 21.9$ & $36.4 \pm 8.2$ & NS \\
\hline
\end{tabular}

Values are presented as mean \pm standard deviation or number (\%).

AEST, aspiration and ethanol sclerotherapy; NS, not significant; BMI, body mass index; AFC, antral follicle count; OR, oocyte retrieval; IVF, in vitro fertilization.

${ }^{a)}$ Significantly lower than in the aspiration or control group $\left(P=0.024, P=0.017\right.$, respectively); ${ }^{b}$ Significantly higher than in the aspiration or control group $(P=0.005, P=0.005)$. 


\section{Obstetrics \& Gynecology Science}

Vol. 57, No. 4, 2014

Table 2. Comparison of controlled ovarian stimulation results and in vitro fertilization/intracytoplasmic sperm injection outcome

\begin{tabular}{|c|c|c|c|c|}
\hline & $\begin{array}{l}\text { Cystectomy group } \\
\qquad(\mathrm{n}=36)\end{array}$ & $\begin{array}{l}\text { AEST group } \\
(n=29)\end{array}$ & $\begin{array}{c}\text { Control group } \\
(n=36)\end{array}$ & $P$-value \\
\hline No. of cycles initiated & 36 & 29 & 36 & - \\
\hline No. of cycles retrieved & 36 & 29 & 36 & - \\
\hline No. of ET cycles & 35 & 27 & 34 & - \\
\hline No. of cycles cancelled & $1(2.7)$ & $2(6.8)$ & $2(5.5)$ & NS \\
\hline No. of GnRH antagonist protocol & $13(36.1)$ & $11(37.9)$ & $13(36.1)$ & NS \\
\hline Days of rhFSH & $11.9 \pm 3.2$ & $12.1 \pm 2.8$ & $12.0 \pm 2.8$ & NS \\
\hline Total dose of rhFSH (IU) & $1,940.2 \pm 407.1$ & $2,015.5 \pm 673.5$ & $1,818.7 \pm 490.1$ & NS \\
\hline \multicolumn{5}{|l|}{ No. of follicles on hCG day } \\
\hline 14 to $<17 \mathrm{~mm}$ & $4.1 \pm 2.1^{a)}$ & $6.1 \pm 3.2$ & $6.2 \pm 3.5$ & 0.003 \\
\hline$\geq 17 \mathrm{~mm}$ & $3.3 \pm 3.0$ & $3.1 \pm 1.4$ & $3.8 \pm 2.0$ & NS \\
\hline EMT on hCG day & $10.2 \pm 1.2$ & $10.3 \pm 1.4$ & $10.0 \pm 1.2$ & NS \\
\hline No. of oocytes retrieved & $8.2 \pm 4.7^{\mathrm{b})}$ & $12.4 \pm 7.6$ & $12.4 \pm 7.5$ & 0.016 \\
\hline No. of mature oocytes & $6.9 \pm 3.7^{c)}$ & $10.5 \pm 6.4$ & $10.7 \pm 6.7$ & 0.010 \\
\hline No. of fertilized oocytes & $5.4 \pm 3.3^{d)}$ & $8.4 \pm 5.3$ & $8.1 \pm 4.8$ & 0.012 \\
\hline No. of grade I, II embryos & $1.8 \pm 1.0$ & $1.7 \pm 0.8$ & $1.7 \pm 1.1$ & NS \\
\hline No. of embryos transferred & $2.7 \pm 1.0$ & $3.2 \pm 1.1$ & $2.9 \pm 0.9$ & NS \\
\hline CPR per cycle initiated (\%) & $36.1(13 / 36)$ & $41.3(12 / 29)$ & $38.8(14 / 36)$ & NS \\
\hline CPR per ET (\%) & $37.1(13 / 35)$ & $44.4(12 / 27)$ & $41.1(14 / 34)$ & NS \\
\hline Miscarriage rate (\%) & $7.6(1 / 13)$ & $8.3(1 / 12)$ & $14.2(2 / 14)$ & NS \\
\hline LBR per cycle initiated (\%) & $33.3(12 / 36)$ & $40.7(11 / 27)$ & $33.3(12 / 36)$ & NS \\
\hline Multiple PR per clinical pregnancy (\%) & $7.6(1 / 13)$ & $8.3(1 / 12)$ & $7.1(1 / 14)$ & NS \\
\hline
\end{tabular}

Values are presented as mean \pm standard deviation or number (\%) unless otherwise indicated.

AEST, aspiration and ethanol sclerotherapy; ET, embryo transfer; NS, not significant; GnRH, gonadotropin releasing hormone; rhFSH, recombinant human follicle stimulating hormone; $h C G$, human chorionic gonadotropin; EMT, endometrial thickness; CPR, clinical pregnancy rate; LBR, live birth rate; PR, pregnancy rate.

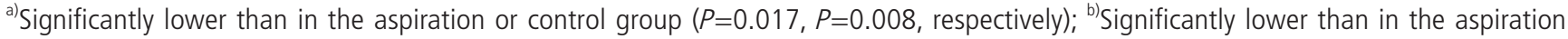
or control group $\left(P=0.05, P=0.031\right.$, respectively); ${ }^{c}$ Significantly lower than in the aspiration or control group $(P=0.042, P=0.018$, respectively); ${ }^{d}$ Significantly lower than in the aspiration or control group $(P=0.028, P=0.037$, respectively).

Analysis of variance test with Bonferroni's post hoc correction was used to compare mean values among three groups while chi-square test and Fisher exact test were used to compare fractions. Statistical significance was defined as $P<0.05$. All analyses were performed by using SPSS ver. 11.0 (SPSS Inc., (hicago, IL, USA).

\section{Results}

The three groups did not differ in terms of the ages of the patients and their spouses, BMI, infertility duration, and the proportion of nullipara. The duration between surgery and $\mathrm{OR}$ was $20.3 \pm 19.5$ months and the size of recurred endometrioma on the day of OR was $22.6 \pm 24.1 \mathrm{~mm}$ in resection group. The duration between AEST and OR was $3.1 \pm 3.0$ months and the size of endometrioma on the day of OR was $15.5 \pm 21.9$ $\mathrm{mm}$ in AEST group. The size of endometrioma on the day of OR in control group was $36.4 \pm 8.2 \mathrm{~mm}$. Total antral follicle count (AFC) was significantly lower in the resection group than in the aspiration or control group $(P=0.007)$. The proportion of patients with AFC $\leq 6$ was significantly higher in the resection group than in other two groups $(P=0.005)$ (Table 1$)$.

In the resection group, 1 out of 36 cycles initiated (2.7\%) was cancelled before ET because no available oocytes were retrieved. Two out of 29 cycles initiated (6.8\%) in the aspi- 


\section{Obstetrics \& Gynecology Science}

Kyung-Hee Lee, et al. Intervention of endometrioma before IVF

ration group and 2 out of 36 cycles initiated (5.5\%) in the control group were also cancelled before ET because available oocytes were not retrieved. There was no significant difference in overall cycle cancellation rate among three groups.

The numbers of 14 to $17 \mathrm{~mm}$ follicles, retrieved oocytes, mature oocytes, fertilized oocytes were significantly lower in the resection group than in other two groups. However, three groups were similar in terms of the number of follicles more than $17 \mathrm{~mm}$ on hCG day, total dose and days of gonadotropin used for COS, the numbers of grade I or II embryos and transferred embryos, clinical pregnancy rate (CPR) per initiated cycle, CPR per ET, embryo implantation rate, miscarriage rate, and multiple pregnancy rate (Table 2).

\section{Discussion}

Endometriosis is a multifactorial disease that seriously compromises the female fertility. The mechanisms by which endometriosis impairs fertility have not been completely proven, but there are some reliable hypotheses. Severe endometriosis is associated with pelvic adhesions and a distortion of pelvic anatomy leading to a possible anatomic disturbance of fertility. In addition, endometriosis may have a direct negative effect on follicular development, oocyte development, embryogenesis, or implantation even in a mild stage. Mediating factors are supposed to be local paracrine action of interleukins or other cytokines, alteration in inflammatory response or autoimmune factors $[1,3]$.

Many treatment have been proposed and applied to overcome the infertility associated with endometriosis. In a meta-analysis of 22 independent studies, Barnhart et al. [2] determined that the overall likelihood of achieving pregnancy rate was significantly lower for stage III-IV endometriosis patients compared with tubal factor control subjects. However, in spite of a lower success rate compared with that of women undergoing IVF for other indications, IVF is still the most successful form of ART that can be offered to an infertile couple with endometriosis. It has already been demonstrated that the presence of endometriosis decreases pregnancy rates for couples who attempt conception without ARTs or with ovulation induction [5-7].

Whether surgical resection improves the IVF outcome is stiil up for debate. Whereas some studies concluded that ovarian cystectomy may be deleterious to residual oocyte and hor- mone function $[5,8,9]$, another study showed no differences in fertility outcomes among patients who underwent the resection of endometrioma, patients with endometriosis without ovarian endometriomas, and patients with tubal infertility [10]. In addition, there are some evidences that ovarian cystectomy for endometriomas should be associated with significantly diminished ovarian reserve after surgery $[11,12]$. A metaanalysis by Somigliana et al. [13] concluded that serum antimüllerian hormone (AMH) level declines after the stripping of ovarian endometrioma and the magnitude of this reduction is more evident in women operated for bilateral cysts. Although the pathogenetic mechanisms mediating the injury to the ovarian reserve remain to be elucidated, the stripping procedure may determine the accidental removal of healthy ovarian tissue [14]. This was analyzed by Kitajima et al. [15] who indicated the presence of normal ovarian tissue in the enucleated cyst as a significant factor influencing the rate of serum $\mathrm{AMH}$ decline. A recent meta-analysis of five studies concluded that surgical management of endometriomas has no significant effect on IVF outcome and ovarian response to COS compared with no treatment [16].

Another option for managing endometrioma is AEST of the endometriotic cyst under transvaginal ultrasound guidance. There are still conflicting data whether this intervention is beneficial for fertility outcome. Guo et al. [17] performed the retrospective comparative study on the pregnancy outcomes of IVF-ET between long-acting GnRH agonist combined with transvaginal ultrasound-guided cyst aspiration and longacting GnRH agonist alone in 134 infertile women. They concluded that serum E2 level on hCG day, the numbers of ovarian follicles of $\geq 14 \mathrm{~mm}$ in diameter and oocytes retrieved, high-quality embryo rate, implantation rate, and CPR were significantly higher in cyst aspisration group than in control group [16]. In a study on the efficacy of aspiration of ovarian endometriomas before ICSI, study subjects were divided into four groups (aspiration of endometriomas at the beginning of COS in patients with ovarian endometriomas with no history of previous surgery, nonaspirated endometriomas with no history of previous surgery, history of ovarian surgery for endometriomas in patients without ovarian endometriomas at the beginning of COS, and tubal factor infertility) [18]. They demonstrated that neither cyst aspiration with or without alcohol fixation nor surgical resection of endometrioma present any beneficial effect of reproductive outcome [18]. One prospective study comparing IVF outcomes with or without 


\title{
Obstetrics \& Gynecology Science
}

\author{
Vol. 57, No. 4, 2014
}

pretreatment in patients with endometrioma demonstrated that neither cyst aspiration nor surgical resection of endometrioma is necessary for ovarian endometrial cyst before IVF-ET. However, they proposed that cyst aspiration may be beneficial after several failed attempts of IVF for slightly increased fertilization rate although it was statistically not significant [19].

In the present study, we investigated the impact of surgical resection and AEST of endometrioma before IVF/ICSI in infertile patients. Our study did not show any beneficial effect of surgical resection and AEST of endometrioma on improving reproductive outcome in IVF cycles. Moreover, surgical resection of endometrioma can affect the ovarian reserve and ovarian response during COS.

However, AEST of endometriomas before IVF may be considerable in patients with large endometrioma(s) interfering with OR because AEST can reduce the volume of endometriomas without decrease of ovarian reserve.

In conclusion, pretreatment including surgical resection and AEST of endometrioma cannot give any beneficial effect before IVF/ICSI. Considering that surgical resection of endometrioma could affect ovarian reserve and ovarian response during COS, resection of endometrioma should be avoided in the patient with low ovarian reserve including elderly women. However, our study has a limitation to evaluate the effect of surgical resection and AEST of endometriomas before IVF due to a small number of sample available and the characteristics of objects were heterogenous due to its retrospective nature. Moreover, there were some limitation to obtain the surgical information because all the patient included in the resection group did not have operation in our hospital. Therefore, welldesigned prospective randomized trials are required to confirm the results of our present study in infertile patients with ovarian endometriomas.

\section{Conflict of interest}

No potential conflict of interest relevant to this article was reported.

\section{References}

1. Ayers JW, Birenbaum DL, Menon KM. Luteal phase dysfunction in endometriosis: elevated progesterone levels in peripheral and ovarian veins during the follicular phase. Fertil Steril 1987;47:925-9.

2. Barnhart K, Dunsmoor-Su R, Coutifaris C. Effect of endometriosis on in vitro fertilization. Fertil Steril 2002;77:1148-55.

3. Hahn DW, Carraher RP, Foldesy RG, McGuire JL. Experimental evidence for failure to implant as a mechanism of infertility associated with endometriosis. Am J Obstet Gynecol 1986;155:1109-13.

4. Benschop L, Farquhar C, van der Poel N, Heineman MJ. Interventions for women with endometrioma prior to assisted reproductive technology. Cochrane Database Syst Rev 2010;(11):CD008571.

5. Falcone T, Goldberg JM, Miller KF. Endometriosis: medical and surgical intervention. Curr Opin Obstet Gynecol 1996;8:178-83.

6. Kodama H, Fukuda J, Karube H, Matsui T, Shimizu Y, Tanaka T. Benefit of in vitro fertilization treatment for endometriosis-associated infertility. Fertil Steril 1996;66:974-9.

7. Pagidas K, Falcone T, Hemmings R, Miron P. Comparison of reoperation for moderate (stage III) and severe (stage IV) endometriosis-related infertility with in vitro fertilization-embryo transfer. Fertil Steril 1996;65:791-5.

8. Benaglia L, Somigliana E, Vighi V, Ragni G, Vercellini P, Fedele L. Rate of severe ovarian damage following surgery for endometriomas. Hum Reprod 2010;25:678-82.

9. Loh FH, Tan AT, Kumar J, Ng SC. Ovarian response after laparoscopic ovarian cystectomy for endometriotic cysts in 132 monitored cycles. Fertil Steril 1999;72:316-21.

10. Canis M, Pouly JL, Tamburro S, Mage G, Wattiez A, Bruhat MA. Ovarian response during IVF-embryo transfer cycles after laparoscopic ovarian cystectomy for endometriotic cysts of $>3 \mathrm{~cm}$ in diameter. Hum Reprod 2001;16:2583-6.

11. Biacchiardi CP, Piane LD, Camanni M, Deltetto F, Delpiano EM, Marchino GL, et al. Laparoscopic stripping of endometriomas negatively affects ovarian follicular reserve even if performed by experienced surgeons. Reprod Biomed Online 2011;23:740-6.

12. Hirokawa W, Iwase A, Goto M, Takikawa S, Nagatomo $Y$, Nakahara T, et al. The post-operative decline in serum anti-Mullerian hormone correlates with the bilaterality and severity of endometriosis. Hum Reprod 2011;26:904-10. 


\section{Obstetrics \& Gynecology Science}

Kyung-Hee Lee, et al. Intervention of endometrioma before IVF

13. Somigliana E, Berlanda N, Benaglia L, Vigano P, Vercellini $P$, Fedele L. Surgical excision of endometriomas and ovarian reserve: a systematic review on serum antimy and severity of endometriosis. Fertil Steril 2012;98:15318.

14. Garcia-Velasco JA, Somigliana E. Management of endometriomas in women requiring IVF: to touch or not to touch. Hum Reprod 2009;24:496-501.

15. Kitajima M, Khan KN, Hiraki K, Inoue T, Fujishita A, Masuzaki $\mathrm{H}$. Changes in serum anti-Müllerian hormone levels may predict damage to residual normal ovarian tissue after laparoscopic surgery for women with ovarian endometrioma. Fertil Steril 2011;95:2589-91.e1.

16. Tsoumpou I, Kyrgiou M, Gelbaya TA, Nardo LG. The effect of surgical treatment for endometrioma on in vitro fertilization outcomes: a systematic review and meta- analysis. Fertil Steril 2009;92:75-87.

17. Guo YH, Lu N, Zhang Y, Su YC, Wang Y, Zhang YL, et al. Comparative study on the pregnancy outcomes of in vitro fertilization-embryo transfer between long-acting gonadotropin-releasing hormone agonist combined with transvaginal ultrasound-guided cyst aspiration and longacting gonadotropin-releasing hormone agonist alone. Contemp Clin Trials 2012;33:1206-10.

18. Pabuccu R, Onalan G, Goktolga U, Kucuk T, Orhon $E$, Ceyhan T. Aspiration of ovarian endometriomas before intracytoplasmic sperm injection. Fertil Steril 2004;82:705-11.

19. Suganuma N, Wakahara Y, Ishida D, Asano M, Kitagawa T, Katsumata $Y$, et al. Pretreatment for ovarian endometrial cyst before in vitro fertilization. Gynecol Obstet Invest 2002;54 Suppl 1:36-40. 